11

\title{
Характеристики поля поверхностной электромагнитной волны на большом расстоянии от излучателя
}

\author{
(C) В.К. Балханов, Ю.Б. Башкуев
}

Институт фризического материаловедения СО РАН

670047 Улан-Удэ, Россия,

e-mail: ballar@yandex.ru

(Поступило в Редакцию 23 марта 2016 г. В окончательной редакции 16 июня 2016 г.)

\begin{abstract}
Представлено приближенное на большом (в длинах волн) расстоянии от излучателя решение задачи Зоммерфельда о поле вертикального электрического диполя, расположенного вблизи границы раздела сред. Установлены характеристики поверхностной электромагнитной волны (ПЭВ), распространяющейся над средой с сильноиндуктивным поверхностным импедансом $\delta$. Пространственные характеристики ПЭВ выражены через действительные и мнимые части импеданса $\delta$. Дано доказательство того, что в электромагнитное поле земной волны для сильноиндуктивных радиотрасс основной вклад дает только ПЭВ.
\end{abstract}

DOI: 10.21883/JTF.2017.04.44322.1817

\section{Введение}

Для описания радиотрассы над земной поверхностью, вдоль которой распространяется электромагнитное поле, часто достаточно знать приведенный поверхностный импеданс $\delta$. Если квадрат модуля импеданса $|\delta|^{2} \ll 1$ и фаза импеданса $\varphi_{\delta}$ лежит в пределах $-45^{\circ}-90^{\circ}$, то радиотрасса называется сильноиндуктивной. Распространение электромагнитного поля вдоль такой сильноиндуктивной радиотрассы приобретает особый характер из-за появления поверхностной электромагнитной волны (ПЭВ). В природе к таким радиотрассам относятся покрытые льдом арктические и антарктические моря Земного шара и соленые озера континентов в зимнее время. Типичным примером является озеро Сульфат на юге Сибири [1]. В зимне-весенний период это озеро является сильноиндуктивной средой. Электромагнитное поле, распространяясь вдоль сильноиндуктивной радиотрассы, оказывается локализованным вблизи поверхности раздела „воздух-импедансная среда“. Поэтому такое поле и называется ПЭВ. Цель насятоящей работы установить структуру ПЭВ на границе раздела, рассмотреть углы наклона фронта волны в воздухе и в среде к импедансной поверхности. Выразить пространственные характеристики ПЭВ через действительные и мнимые части импеданса $\delta$. В отличие от метода Зоммерфельда для определения компонент ПЭВ решалось непосредственно волновое уравнение (уравнение Гельмгольца), причем решение искалось в волновой зоне - на расстояниях, больших, чем длина волны. Дадим доказательство, что в электромагнитное поле земной волны для сильноиндуктивных радиотрасс основной вклад дает только ПЭВ.

Существование ПЭВ впервые более 100 лет назад предположил А. Зоммерфельд. Для существования ПЭВ необходимы два условия. Во-первых, электромагнитная волна должна быть вертикально поляризованной, т.е. ее излучателем является вертикальная относительно зем- ной поверхности электрическая антенна. Во-вторых, подстилающая среда должна обладать сильноиндуктивным поверхностным импедансом [2,3]. Поля, переносимые вдоль такой радиотрассы, локализованы вблизи поверхности, т.е. затухают в воздухе и в импедансной среде. Электрический вектор Е ПЭВ имеет две составляющие: радиальную $E_{r}$ - вдоль поверхности $z=0$ и вертикальную $E_{z}-$ нормально поверхности; магнитное поле $H_{y}$ перпендикулярно направлению распространения волны и лежит в плоскости поверхности.

Мы выбираем цилиндрическую систему координат, такую, что радиальная ось $r$ лежит на поверхности, ось $z$ направлена по нормали к поверхности в воздух. Введем вектор $\mathbf{k}$ направления фронта волны. Его квадрат $k^{2}$, согласно уравнениям Максвелла [4]:

$$
k^{2}=\frac{\omega^{2}}{c^{2}} \varepsilon+\frac{i \mu_{0} \omega}{\rho},
$$

где $\omega-$ круговая частота, $c-$ скорость света, $\varepsilon-$ относительная диэлектрическая проницаемость среды (для воздушной среды $\varepsilon=1), \rho-$ удельное электрическое сопротивление вещества (для воздуха можно полагать $\rho \rightarrow \infty), \mu_{0}$ - магнитная постоянная.

Распространение поля происходит вдоль радиальной координаты $r$, вектор магнитного поля Н вблизи земной поверхности имеет только одну ненулевую компоненту $H_{y}(r, z)$ :

$$
\mathbf{H}=\left(0, H_{y}(r, z), 0\right) .
$$

В цилиндрической системе координат компоненты электрического поля будут находиться из следующих выражений:

$$
E_{r}=-\frac{i \mu_{0} \omega}{k^{2}} \frac{\partial}{\partial z} H_{y}, \quad E_{z}=\frac{i \mu_{0} \omega}{k^{2}} \frac{1}{r} \frac{\partial}{\partial r}\left(r H_{y}\right) .
$$

Временна́я зависимость дается множителем $\exp (-i \omega t)$, она одинакова для всех компонент полей и нами не 
выписывается. Введем поверхностный импеданс $\delta$ следующим выражением [4]:

$$
\delta=-\frac{1}{\mu_{0} c}\left(\frac{E_{r}}{H_{y}}\right)_{z=0} .
$$

Введение импеданса соотношением (4) называется условием Леонтовича. Его квадрат модуля оказывается много меньше единицы. Это необходимо, чтобы иметь дело с переменными полями. Знак минус в (4) связан с выбором ориентации оси $z$. Если бы ось $z$ ориентировалась вглубь среды, то в (4) был бы знак „+“. Электрические свойства подстилающей среды полностью описываются импедансом $\delta$. Согласно книге Е.Л. Фейнберга [5], „Среды, с которыми приходится иметь дело в теории распространения радиоволн вдоль земли, это почва и атмосфера“. И далее „... поверхностный слой земли всегда ведет себя совершенно различно в отношении волн различных частот“" [5]. Если $\mu_{0} \omega / \rho \gg \omega^{2} \varepsilon / c^{2}$, то такие среды называются индуктивными или импедансными. Импеданс $\delta$ является комплексной величиной, и его записывают как $\delta=|\delta| \exp (i \varphi)_{\delta}$, где $|\delta|-$ модуль и $\varphi_{\delta}-$ фаза импеданса. Если фаза $\varphi_{\delta}$ имеет значение чуть больше, чем $-90^{\circ}$, то соответствующие им среды называются сильноиндуктивными. К таким средам на Земном шаре относятся покрытые льдом арктические и антарктические моря и соленые озера континентов в зимне-весеннее время.

В цилиндрической системе координат при полной угловой симметрии волновое уравнение для магнитного поля принимает следующий вид:

$$
\frac{1}{r} \frac{\partial}{\partial r}\left(r \frac{\partial H_{y}}{\partial r}\right)+\frac{\partial^{2}}{\partial} \frac{H_{y}}{z^{2}}+k^{2} H_{y}=0 .
$$

Начиная с А. Зоммерфельда, решение подобного уравнения для задач распространения радиоволн обычно сводят к комплексному интегралу, вычисление которого вызывает определенные трудности [6].

Однако в 80-х годах прошлого века И. Линделлом и его коллегами предложен метод мнимых изображений применительно к задачам Зоммерфельда [7]. Решение при этом также записывается в виде комплексных интегралов, но сходимость которых на несколько порядков лучше, что позволяет существенно повысить скорость их вычисления. Мы же уравнение (5) будем решать методом разделения переменных. На расстояниях $r$ таких, что

$$
r \gg \frac{1}{|\chi|},
$$

выражениями, содержащими множители вида $\frac{1}{r}, \frac{1}{r^{3 / 2}}$ и т.д., можно пренебречь. Здесь $\chi$ является параметром разделения переменных уравнения (5). Тогда общее решение уравнения (5) в цилиндрической системе координат будет иметь следующий вид:

$$
H_{y}(r, z)=K \frac{1}{\sqrt{r}} \exp \left(i \chi r \pm i \sqrt{k^{2}-\chi^{2}} z\right) .
$$

Решение легко проверяется прямой подстановкой выражения (7) в уравнение (5) и учетом неравенства (6). Если распространение волны происходит в однородной среде и нет никаких границ, то в (7) достаточно выбрать один какой-то знак. Однако если распространение происходит в двухслойной среде, то понадобятся уже два выражения типа (7) с разными знаками.

По порядку величин параметр $\chi$ совпадает с обратной величиной длины волны (в воздухе), так что условие (6) совпадает с понятием волновой зоны - поле рассматривается на расстояниях от излучателя много больших, чем длина волны. Этим самым мы ищем решение в волновой зоне. Как отмечено в $[5]$, „. . в волновой зоне можно ... пренебречь волной, доходящей от источника через почву“. И там же: для выполнения „граничных условий ... достаточно рассмотреть поля на малом участке поверхности, лишь бы на нем укладывалось несколько длин волн““. Последнее всегда выполняется для вертикально ориентированной относительно поверхности земли приемной электрической антенны.

\section{Структура поля на границе раздела}

Мы рассматриваем область воздух-сильно индуктивная среда. Величины, относящиеся к воздуху, будем маркировать индексом 0 , для среды величины будут без маркера. С учетом нашего выбора ориентации оси $z$ выпишем магнитное поле в среде

$$
H_{y}(r, z)=K \frac{1}{\sqrt{r}} \exp \left(i \chi r-i \sqrt{k^{2}-\chi^{2}} z\right) .
$$

Знак „-““ при координате $z$ выбирался из условия, чтобы при распространении вглубь среды ПЭВ испытывала затухание. Компоненты электрического поля с учетом неравенства (6) находятся из выражений (3).

Из известных граничных условий о том, что тангенциальные компоненты полей на границе воздух-среда одинаковы, следует неизменность постоянных $K$ и $\chi$ в обеих средах (Приложение). Таким образом, в воздухе

$$
H_{0 y}(r, z)=K \frac{1}{\sqrt{r}} \exp \left(i \chi r-i \sqrt{k_{0}^{2}-\chi^{2}} z\right) .
$$

Здесь квадрат волнового числа в воздухе $k_{0}^{2}=\omega^{2} / c^{2}$. Далее из граничных условий следует

$$
\delta=\frac{1}{\sqrt{1+\left(k / k_{0}\right)^{2}}} .
$$

Из (1) и (10) видно, что действительная часть импеданса $\operatorname{Re} \delta>0$, а мнимая часть $\operatorname{Im} \delta=-|\operatorname{Im} \delta|<0$.

Далее находим

$$
\chi=k_{0} \sqrt{1-\delta^{2}} .
$$

При выполнении неравенства

$$
|\delta|^{2} \ll 1
$$


точное выражение $k=k_{0} \sqrt{-1+1 / \delta^{2}}$, получаемое из (10), принимает следующее приближенное выражение:

$$
k \approx k_{0} / \delta .
$$

Этим самым мы выразили волновое число в среде через волновое число в воздухе и приведенный поверхностный импеданс индуктивной среды.

С учетом полученных результатов магнитное поле в среде и в воздухе можно записать как

$$
\begin{aligned}
& H_{y}(r, z)=K \frac{1}{\sqrt{r}} \exp \left(i \frac{k_{0} k}{\sqrt{k_{0}^{2}+k^{2}}} r-i \frac{k^{2}}{\sqrt{k_{0}^{2}+k^{2}}} z\right), \\
& H_{0 y}(r, z)=K \frac{1}{\sqrt{r}} \exp \left(i \frac{k_{0} k}{\sqrt{k_{0}^{2}+k^{2}}} r-i \frac{k_{0}^{2}}{\sqrt{k_{0}^{2}+k^{2}}} z\right) .
\end{aligned}
$$

Из (3) находим компоненты электрического поля в среде

$$
\begin{gathered}
E_{z}(r, z)=-\frac{\mu_{0} \omega^{2}}{c k \sqrt{k_{0}^{2}+k^{2}}} H_{y}(r, z), \\
E_{r}(r, z)=-\frac{\mu_{0} \omega}{\sqrt{k_{0}^{2}+k^{2}}} H_{y}(r, z)
\end{gathered}
$$

и в воздухе:

$$
\begin{aligned}
& E_{0 z}(r, z)=-\frac{\mu_{0} c k}{\sqrt{k_{0}^{2}+k^{2}}} H_{0 y}(r, z), \\
& E_{0 r}(r, z)=-\frac{\mu_{0} \omega}{\sqrt{k_{0}^{2}+k^{2}}} H_{0 y}(r, z) .
\end{aligned}
$$

Этим самым формулами (14)-(19) описывается структура ПЭВ как в воздухе, так и в импедансной среде. При вычислении $E_{r}(r, z)$ и $E_{0 r}(r, z)$, согласно (6), пренебрегали $1 / 2 r$ по сравнению с $\chi$. Наше рассмотрение согласуется с тем, что „... граничные условия Леонтовича оправдываются всегда, пока можно пренебречь производными от $1 / r \ldots$ Это всегда возможно в волновой зоне“ [5]. В работе [5] для решения волнового уравнения вводилась промежуточная функция - функция ослабления, для которой методом функции Грина находилось интегральное уравнение. Наше рассмотрение отличается тем, что мы сразу искали решение дифференциального уравнения (5) с учетом выполнения неравенства (6).

Далее обратим внимание, что поскольку $|\delta|^{2} \ll 1$, то магнитное поле в воздухе можно представить как

$$
H_{0 y}(r, z)=K \frac{1}{\sqrt{r}} \exp \left(i k_{0} r-i k_{0} \frac{\delta^{2}}{2} r-i k_{0} \delta z\right) .
$$

Оно совпадает с известным решением Зоммерфельда для ПЭВ, полученным им в волновой зоне из интегрального уравнения для функции ослабления на проводящей радиотрассе. В импедансной среде магнитное поле будет

$$
H_{y}(r, z)=K \frac{1}{\sqrt{r}} \exp \left(i k_{0} r-i k_{0} \frac{\delta^{2}}{2} r-i \frac{k_{0}}{\delta} z\right) .
$$

Найдем углы наклона волнового фронта ПЭВ к плоскости распространения. Эти углы $\varphi_{0}$ и $\varphi$ в первом и четвертом квадрантах соответственно определяются как в воздухе

$$
\operatorname{tg} \varphi_{0}=\frac{E_{0 z}}{E_{0 r}}
$$

в импедансной среде

$$
\operatorname{tg} \varphi=\frac{\left|E_{z}\right|}{\left|E_{r}\right|}
$$

Используя (16)-(19), находим

$$
\operatorname{tg} \varphi_{0}=\frac{|k|}{k_{0}}, \quad \operatorname{tg} \varphi=\frac{k_{0}}{|k|} .
$$

Или с учетом (13)

$$
\operatorname{tg} \varphi_{0}=\frac{1}{|\delta|}, \quad \operatorname{tg} \varphi=|\delta| .
$$

Поскольку выполняется неравенство (12), то $\varphi_{0} \approx$ $\approx 90^{\circ}$, - в воздухе волна распространяется вдоль поверхности. В сильноиндуктивной импедансной среде $\varphi \approx 0^{\circ}$, и волна распространяется вглубь среды. Кроме того, из (24) следует, что углы $\varphi$ и $\varphi_{0}$ совместно составляют прямой угол. Из (20) и (21) следует, что ПЭВ в воздухе по высоте и ПЭВ вглубь импедансной среды затухают, что и означает локализацию электромагнитного поля вблизи поверхности (появление ПЭВ). Поскольку выполняется неравенство (12), то в воздухе ПЭВ затухает по высоте на расстоянии в несколько длин волн. В импедансной же среде затухание происходит уже на длине волны. В радиальном направлении вдоль поверхности амплитуда ПЭВ затухает следующим образом: $\frac{K}{\sqrt{r}} \exp (-B r)$. А это заметно медленнее, чем если бы распространение происходило вдоль идеально проводящей поверхности, где пространственное затухание происходит как $1 / r$.

Слабое затухание ПЭВ по сравнению с другими волнами и ее локализация вблизи границы раздела в последние два десятка лет начали находить широкое применение для различных технических устройств, например [8-10]. ПЭВ также нашли широкое применение для целей радиолокации. Они найдут свое применение для установления дальней радиосвязи в районах Крайнего Севера и Северного Ледовитого океана. Также, например, с помощью ПЭВ можно определять климатические изменения в Северном Ледовитом океане без привлечения спутниковой локации.

\section{Пространственные характеристики}

Углы $\varphi_{0}$ и $\varphi$ выражены через модуль импеданса сильноиндуктивной подстилающей среды. Также через импеданс можно выразить пространственные характеристики компонентов полей. 


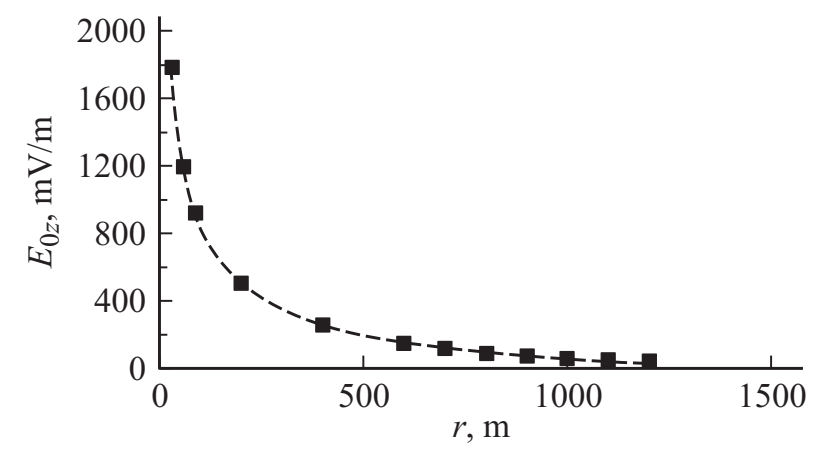

Квадратные маркеры - результаты измерений. Штриховая линия - вычисление по формуле $(33)$ при $z=0$. Излучаемая мощность $1 \mathrm{kWatt}$.

Беря абсолютные значения компонентов полей (14)-(19), находим следующие пространственные характеристики для магнитного поля:

$$
\begin{aligned}
& \left|H_{y}(r, z)\right|=K \frac{1}{\sqrt{r}} \exp \left(-k_{0} \operatorname{Re} \delta|\operatorname{Im} \delta| r+\frac{k_{0}|\operatorname{Im} \delta|}{|\delta|^{2}} z\right) \\
& \left|H_{0 y}(r, z)\right|=K \frac{1}{\sqrt{r}} \exp \left(-k_{0} \operatorname{Re} \delta|\operatorname{Im} \delta| r-k_{0}|\operatorname{Im} \delta| z\right)
\end{aligned}
$$

и для компонент электрического поля

$$
\begin{gathered}
\left|E_{z}(r, z)\right|=-\mu_{0} c|\delta|^{2}\left|H_{y}(r, z)\right|, \\
\left|E_{r}(r, z)\right|=-\mu_{0} c|\delta|\left|H_{y}(r, z)\right|, \\
\left|E_{0 z}(r, z)\right|=-\mu_{0} c\left|H_{0 y}(r, z)\right|, \\
\left|E_{0 r}(r, z)\right|=-\mu_{0} c|\delta|\left|H_{0 y}(r, z)\right| .
\end{gathered}
$$

Выпишем выражение для пространственной характеристики вертикальной компоненты электрического поля в свободном пространстве:

$$
\left|E_{0 z}(r, z)\right|=K \frac{1}{\sqrt{r}} \exp \left(-k_{0} \operatorname{Re} \delta|\operatorname{Im} \delta| r-k_{0}|\operatorname{Im} \delta| z\right) .
$$

При этом из сравнения с решением Зоммерфельда [5] можно выразить множитель $K$ через излучаемую мощность $P\left(10^{3} \mathrm{~W}\right)$, а именно

$$
K=\frac{300 \sqrt{P}}{\mu_{0} c} .
$$

Само электрическое поле измеряется в $10^{-3} \mathrm{~V} / \mathrm{m}$.

\section{Озеро Сульфат}

В ходе натурных измерений в зимнее время на озере Сульфат [1] на частоте $f=10 \mathrm{MHz}$ была установлена пространственная характеристика вертикальной компоненты электрического поля. Измеренный ход зависимости $E_{0 z}(r, z=0)=\frac{K}{\sqrt{r}} \exp (-B r)$ показан на рисунке маркерами в виде квадратов. Здесь множитель $K$ дается выражением (33), коэффициент $B=k_{0} \operatorname{Re} \delta|\operatorname{Im}| \delta$. Численные значения $K=10273$ и $B=0.00178$ (подробности вычислений даны в [11]). При этих значениях $K$ и $B$ зависимость $E_{0 z}(r, z=0)$ представлена на рисунке пунктирной линией. Наблюдаем более чем удовлетворительное совпадение. Кроме того, в работе [11] показано, как по установленным значениям для $K$ и $B$ находятся модуль и фаза поверхностного импеданса. Установлены $|\delta|=0.153$ и фаза $\psi_{\delta}=-65.5^{\circ}$. Полученные значения удовлетворяют условию Леонтовича $|\delta|^{2} \ll 1$ и условию $-45.1^{\circ}>\psi_{\delta}>-89.90$, характерным для появления ПЭВ.

Распространяющиеся вдоль земной поверхности электромагнитные волны называются полем земной волны. Поле земной волны состоит из суперпозиции различных полей, и, как показывает совпадение обеих кривых на рисунке, из всей совокупности полей для сильноиндуктивных радиотрасс преобладающей является ПЭВ. В литературе имеется большое количество монографий и учебных пособий по распространению радиоволн, например [12-14]. Поэтому мы только конспективно дадим обоснование того, что поле земной волны для сильноиндуктивных радиотрасс состоит в основном из ПЭВ.

Для поля земной волны над плоской поверхностью вектор Герца Г состоит из двух слагаемых

$$
\Gamma=\Gamma_{1}+\Gamma_{2}
$$

Разделение поля земной волны на слагаемые связано только с математическим удобством. Каждое из них имеет свою пространственную характеристику, для которых удалось получить обозримые математические выражения. Первое слагаемое $\Gamma_{1}$ описывает пространственную или объемную волну и записывается как

$$
\Gamma_{1}=T\left(\frac{\exp \left(i k_{0} r_{1}\right)}{r_{1}}+\frac{\exp \left(i k_{0} r_{2}\right)}{r_{2}} \frac{\delta \operatorname{tg} y-1}{\delta \operatorname{tg} y+1}\right),
$$

где угол скольжения $\gamma$ определяется как $\operatorname{tg} y=\left(h_{1}+\right.$ $\left.+h_{2}\right) / r_{2}$. Здесь $r_{1}$ и $r_{2}-$ расстояния от излучателя и его изображения до приемной антенны, $\delta$ - импеданс подстилающей среды, $h_{1}$ и $h_{2}-$ высоты поднятия излучателя и приемника над поверхностью земли, $T$ - некоторая постоянная. Если расстояние $R$ от излучателя до приемника очень большое, существенно больше длины волны, то величины $r_{1}$ и $r_{2}$ будут мало отличаться от $R$. Кроме того, на таком большом расстоянии поднятие антенн будет совсем незаметно, и можно положить $h_{1}=0$ и $h_{2}=0$. В этом случае видим, что $\Gamma_{1}$ обращается в нуль - пространственная волна почти не доходит до приемника.

Второе слагаемое $\Gamma_{2}$ описывает локализованную вблизи поверхности волну и записывается как

$$
\Gamma_{2} \sim \frac{1}{\sqrt{r_{1}}} \exp \left(-i k_{0} \frac{\delta^{2}}{2} r_{1}\right)
$$

а это и есть ПЭВ. 
Имеется еще боковая волна, распространяющаяся в среде. Но в волновой зоне и для приподнятых над поверхностью антенн она играет малую роль. Если учитывать кривизну поверхности, то в вектор Герца Г добавлялось бы дифракционное слагаемое, которое отсутствует в рассматриваемом нами случае плоской поверхности. Так что из всей совокупности полей, составляющих поле земной волны, для сильноиндуктивных радиотрасс над плоской поверхностью заметный вклад в принимаемое электромагнитное поле вертикально ориентированной антенной дает только ПЭВ.

\section{Заключение}

Для областей, далеких от излучателя, методом разделения переменных предложено решение волнового уравнения. Предложенный метод существенно проще метода Зоммерфельда, который сводится к комплексному интегралу, полное вычисление которого вызывает большие математические трудности. Установлена структура ПЭВ на границе раздела „воздух-сильноиндуктивная среда“. Решена задача выражения пространственной характеристики компонент ПЭВ через импеданс сильноиндуктивной среды, а также найдены углы наклона фронта ПЭВ к поверхности в воздухе и в импедансной среде. В качестве примера рассмотрены результаты натурных измерений электромагнитного поля на озере Сульфат. Доказано, что для сильноиндуктивных радиотрасс в волновой зоне в поле земной волны основной вклад вносит только ПЭВ.

Авторы выражают искреннюю благодарность рецензенту, замечания которого способствовали существенному улучшению статьи.

Исследование выполнено за счет гранта Российского научного фонда (проект № 14-19-01079) в Институте физического материаловедения Сибирского Отделения PAH.

\section{Приложение. Следствия граничных условий}

Имеем ненулевые горизонтальные компоненты магнитного поля в воздухе

$$
H_{0 y}(r, z)=K_{0} \frac{1}{\sqrt{r}} \exp \left(i \chi_{0} r-i \sqrt{k_{0}^{2}-\chi_{0}^{2}} z\right)
$$

и в среде

$$
H_{y}(r, z)=K_{0} \frac{1}{\sqrt{r}} \exp \left(i \chi r-i \sqrt{k^{2}-\chi^{2}} z\right) .
$$

На поверхности при $z=0$ эти поля будут равны друг другу

$$
K_{0} \frac{1}{\sqrt{r}} \exp \left(i \chi_{0} r\right)=K \frac{1}{\sqrt{r}} \exp (i \chi r)
$$

Чтобы это выражение не зависело от координаты $r$, необходимо положить $\chi_{0}=\chi$. Тогда отсюда следует, что и $K_{0}=K$.

Из (3) с учетом того, что $\chi_{0}=\chi$ и $K_{0}=K$, находим горизонтальные компоненты электрического поля

$$
\begin{aligned}
E_{0 r}(r, z)= & -\mu_{0} \omega \frac{\sqrt{k_{0}^{2}-\chi^{2}}}{k_{0}^{2}} K \frac{1}{\sqrt{r}} \\
& \times \exp \left(i \chi r-i \sqrt{k_{0}^{2}-\chi^{2} z}\right), \\
E_{r}(r, z)= & -\mu_{0} \omega \frac{\sqrt{k^{2}-\chi^{2}}}{k_{0}^{2}} K \frac{1}{\sqrt{r}} \\
& \times \exp \left(i \chi r-i \sqrt{k^{2}-\chi^{2} z}\right) .
\end{aligned}
$$

Эти поля должны быть равны друг другу при $z=0$, откуда следует $\frac{\omega}{c k_{0}^{2}} \sqrt{k_{0}^{2}-\chi^{2}}=\frac{\omega}{c k^{2}} \sqrt{k^{2}-\chi^{2}}$. Отсюда $\chi^{2}=\frac{k_{0}^{2} k^{2}}{k_{0}^{2}+k^{2}}$. Подставляя поля $E_{r}(r, z)$ и $H_{y}(r, z)$ в (4), далее находим $\delta=\frac{\sqrt{k_{0}^{2}-\chi^{2}}}{k_{0}}=\frac{1}{\sqrt{1+\left(k / k_{0}\right)^{2}}}$, т.е. $(10)$, а также $\chi=k_{0} \sqrt{1-\delta^{2}}$, т.е. (11).

\section{Список литературы}

[1] Башкуев Ю.Б., Хаптанов В.Б., Дембелов М.Г. // Письма в ЖТФ. 2010. Т. 36. Вып. 3. С. 88-95.

[2] Макаров Г.И., Новиков В.В., Рыбачек С.Т. Распространение электромагнитных волн над земной поверхностью. М.: Наука, 1991. 196 с.

[3] Цыдыпов Ч.Ц., Цыденов В.Д., Башкуев Ю.Б. Исследование электрических свойств подстилающей среды. Новосибирск: Наук, 1979. 176 с.

[4] Балханов В.К., Башкуев Ю.Б. Основы теории метода поверхностного импеданса. Улан-Удэ: Изд-во Бурятского научного центра СО РАН, 2005. $100 \mathrm{c}$.

[5] Фейнберг Е.Л. Распространение радиоволн вдоль земной поверхности. М.: Физматлит, 1999. 496 с.

[6] http://elib.psuti.ru/part_11.pdf

[7] Lindell I.V., Alanen E. // IEEE Trans. Antenn. Propag. 1984. Vol. AP-32. N 8. P. 841-847.

[8] Аверков Ю.О., Яковенко В.М. // ЖТФ. 2011. Т. 81. Вып. 4. C. $71-77$.

[9] Князев Б.А., Кузьмин А.В. // Вестник НГУ. Сер. физ. 2007. T. 2. Вып. 1. С. 108-122.

[10] Булгаков А.А., Федорин И.В. // ФТТ. 2012. Т. 54. Вып. 8. C. $1470-1477$.

[11] Балханов В.К., Башкуев Ю.Б., Ангархаева Л.Х. // ГИАБ. 2015. № 12. С. 281-284. Электронный ресурс. Режим доступа:

http://www.giab-online.ru/files/Data/2015/12/281_285_12_2015.pdf

[12] Гавриленко В.Г., Яшнов В.А. Распространение радиоволн в современных системах мобильной связи. Нижний Новгород: Изд-во НГУ, 2003. 148 с.

[13] Родос Л.Я. Электродинамика и распространение радиоволн: учебное пособие. СПб.: Изд-во СЗТУ, 2007. 89 с.

[14] Электронный ресурс доступа: http://rudocs.exdat.com/docs/index-75923.html 